\title{
Narrativa
}

\section{Stregati dalla migrazione. Intorno all'esordio di Elvis Malaj}

Ugo Fracassa

\section{(2) OpenEdition}

\section{Journals}

Edizione digitale

URL: https://journals.openedition.org/narrativa/356

DOI: $10.4000 /$ narrativa.356

ISSN: 2804-1224

\section{Editore}

Presses universitaires de Paris Nanterre

\section{Edizione cartacea}

Data di pubblicazione: 1 décembre 2019

Paginazione: 79-90

ISBN: 978-2-84016-350-3

ISSN: $1166-3243$

Notizia bibliografica digitale

Ugo Fracassa, «Stregati dalla migrazione. Intorno all'esordio di Elvis Malaj», Narrativa [Online], 41 |

2019, online dal 01 novembre 2021, consultato il 08 décembre 2021. URL: http://

journals.openedition.org/narrativa/356 ; DOI: https://doi.org/10.4000/narrativa.356

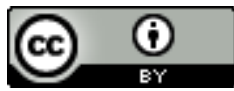

Narrativa est mise à disposition selon les termes de la Licence Creative Commons Attribution 4.0 International. 


\title{
Stregati dalla migrazione. Intorno all'esordio di Evis Malaj
}

\begin{abstract}
Riassunto
Il libro d'esordio di Elvis Malaj ripropone il paradosso dell'italianità di un autore che, per essere nato altrove, rischia di essere letto esclusivamente secondo le categorie della cosiddetta letteratura migrante. Tale paradosso Malaj lo mette in scena con grande consapevolezza nelle 12 storie che compongono la raccolta di racconti Dal tuo terrazzo si vede casa mia. Grazie all'uso dell'ironia e all'abilità nel proporre inserti nella lingua d'origine senza compromettere la comprensibilità del narrato, lo scrittore nativo di Malësi e Madhe, regione albanese, svela l'inconsistenza dei luoghi comuni su/ tra i nuovi italiani.
\end{abstract}

\section{RÉSUMÉ}

Le premier livre de Elvis Malaj nous propose un paradoxe classique: celui d'un auteur italien que l'on perçoit, du fait de ses origines, comme un écrivain migrant. Au fil des 12 histoires qui composent son recueil, Malaj met en place ce paradoxe avec une subtilité remarquable. Grâce au dispositif ironique et à l'insertion de paragraphes en langue étrangère (qui ne compromettent pas la lisibilité du récit), l'écrivain né dans la région albanaise de Malësi et Madhe révèle l'inconsistance des stéréotypes portant sur les « nouveaux Italiens » et répandus parmi eux.

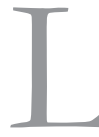

a fragorosa attualità del fenomeno migratorio e della sua controversa gestione da parte del governo italiano, ha fatto irruzione tra gli Amici della Domenica, nel Ninfeo di Villa Giulia, sotto forma di una lettera aperta firmata dai finalisti in occasione della settantaduesima edizione del Premio Strega: "in questi giorni non abbiamo potuto fare a meno di seguire con angoscia la vicenda dei migranti soccorsi a bordo dell'Aquarius dopo che il governo le ha sbarrato l'attracco nei nostri porti [...] Per questi motivi, abbiamo 
unito le nostre voci'”. Con ciò l'edizione del 2018 ha portato all'ordine del giorno anche una questione non più rinviabile nell'agenda del dibattito culturale interno alle "patrie lettere". Di cosa parliamo quando parliamo di letteratura della migrazione? Si tratta di una determinazione anagrafica, di una classificazione tematica, di un'interpretazione critica? Almeno tre libri selezionati tra i dodici finalisti nel 2018 sembrano giustificare un simile interrogativo: i drammatici sbarchi sulle sponde meridionali della penisola di imbarcazioni di fortuna salpate dalle coste del nord Africa forniscono l'innesco narrativo in Da un altro mondo, il fortunato romanzo della palermitana Evelina Santangelo, originale nel declinare un tema usurato dalla cronaca in un registro fantastico-allegorico ("dopo l'ennesimo salvataggio in mare di un numero imprecisato di profughi, migranti, o che diavolo erano..." $)^{2}$; Helena Janeczeck, che si è infine aggiudicata la vittoria, è autrice translingue, essendo arrivata in Italia nel 1983 ed avendo esordito sei anni dopo con una raccolta di versi in tedesco. La storica protagonista del suo La ragazza con la Leica, la fotografa Gerda Taro, ha condiviso con la scrittrice l'esperienza dell'espatrio e la comune provenienza da una famiglia di ebrei polacchi; Dal tuo terrazzo si vede casa mia, il libro di racconti dell'esordiente Elvis Malaj (Malësi e Madhe, 1990), presentato dalla casa editrice Racconti come "primo autore italiano" in collana, rischia infine di essere il maggiore indiziato per una ricezione che si contenga entro la stringente cornice critica della migrazione. Se, infatti, la scrittrice autoctona (è il caso di Santangelo) si sottrae "naturalmente", per diritto di nascita, alla classificazione in uso per autori nati altrove, anche quando non interessati a raccontare la migrazione, un'autrice come la Janeczeck nella logica dell'industria culturale risulta intuitivamente associabile ad una élite letteraria transnazionale per la quale, tradizionalmente, categorie come diaspora, espatrio o "dispatrio"3 risultano più appropriate a descrivere una condizione cosmopolita. L'etichetta di "scrittore migrante" appare dunque, a quasi trent'anni dalla sua acquisizione nel gergo critico italiano, irrevocabilmente inquinata da sottintesi etnici o di classe, indotta da determinazioni economiche e biopolitiche, non diversamente dallo stigma di "emigrante"

1. La lettera è stata pubblicata a pagina 7 del quotidiano la Repubblica il 18 giugno 2018 a firma di Marco Balzano, Carlo Carabba, Carlo D’Amicis, Silvia Ferreri, Helena Janeczeck, Lia Levi, Elvis Malaj, Francesca Melandri, Angela Nanetti, Sandra Petrignani, Andrea Pomella e Yari Selvetella.

2. È quanto si legge nel primo paragrafo del romanzo risultato "libro dell'anno" in un sondaggio condotto tra gli ascoltatori della trasmissione Fahrenheit su Radio 3.

3. Con questo neologismo Luigi Meneghello ha sintetizzato la propria vicenda esistenziale in Il dispatrio, pubblicato da Rizzoli nel 1993. 
attribuito al giovane partenopeo desideroso di viaggiare, reso celebre nel film d'esordio di Massimo Troisi'. Per ragioni uguali e contrarie, la manifestazione in numerosi titoli recenti di scrittori italiani (Fabio Geda, Giuseppe Catozzella, Paolo Di Stefano tra gli altri) di peculiari tratti di genere, quali l'uso della prima persona, la tematizzazione del viaggio e del percorso di integrazione nella società d'arrivo da parte di un personaggio immigrato, la veridicità del narrato, l'urgenza testimoniale del dettato, non produce per questi ultimi l'attribuzione al corpus della letteratura migrante che pure, nella sua prima stagione, aveva fissato quegli stilemi ${ }^{5}$.

La presenza di un giovane autore di origine albanese nella dozzina dello Strega non costituisce peraltro una novità assoluta per il più prestigioso premio letterario nazionale (l'esordio stesso non ha costituito un tabù per i giurati che per quattro volte hanno premiato un romanziere "principiante", come nel caso più recente di Paolo Giordano). Già nel 2005 Ron Kubati era riuscito ad issarsi fino alla cinquina dei papabili con un breve romanzo intitolato Il buio del mare, edito da Giunti. Mentre però in quel caso la narrazione precipitava, sullo sfondo della feroce repressione degli oppositori politici sotto il regime comunista di Enver Hoxha, verso uno scioglimento canonico (il giovanissimo protagonista si imbarca da clandestino per attraversare l'Adriatico e va incontro al suo destino italiano, non prima di aver rinnegato il proprio nome albanese a favore di un beneaugurante Luca), i dodici racconti licenziati da Malaj propongono vicende di varia ambientazione senza mai focalizzare esplicitamente l'immigrazione come tema. Trasferitosi quindicenne in Italia via mare nel 2005, ma in condizioni ben diverse da coloro che avevano affrontato il viaggio stipati sulla nave Vlora salpata da Durazzo quattordici anni prima, l'autore di Dal tuo terrazzo si vede casa mia si è comunque trovato ad occupare, tra gli altri finalisti, una posizione decentrata. Pubblicato nel 2018 da una piccola casa editrice, la romana Racconti, nata solo due anni prima, il suo libro, oltre a segnare un esordio assoluto nel campo della narrativa, non può ambire allo statuto di romanzo, sia pure breve, presentandosi come raccolta di short stories. La candidatura di Malaj ha trovato tuttavia un autorevole promotore nella persona di Luca Formenton,

4. Ricomincio da tre, film prodotto e distribuito in Italia nel 1981.

5. Per l'analisi di questa recente produzione narrativa mi permetto di rimandare il lettore al mio: "Nuove frontiere della letteratura italiana della migrazione", in Scritture migranti, n. 11-12, 2017-2018, pp. 231-266. 
nipote di Arnoldo Mondadori e presidente de Il Saggiatore, che nella sua lettera di segnalazione agli Amici della Domenica non manca di sottolineare il côté multiculturalista dell'operazione:

Ho salutato con interesse la scelta di Racconti edizioni, una piccola casa editrice romana, di puntare su uno scrittore esordiente a cavallo tra due identità, quella albanese e quella italiana, come loro primo autore compiutamente italiano. Trovo salutare che in un paese in cui la legge sullo ius soli è rimasta impantanata nelle secche delle camere, i nuovi scrittori italiani non facciano più di cognome solo Rossi o Bianchi, ma Malaj, Scego, Brahimi [sic], Vorpsi, Lakhous; che a vincere il festival di Sanremo sia Ermal Meta e i ragazzi ascoltino rapper che di nome fanno Ghali'.

In questo apparente terzo grado di minorità (anagrafica, editoriale e di genere), l'adozione della forma racconto mantiene un suo peso specifico. "Esordire con un esordiente, in un mercato come il nostro, con una raccolta di racconti, prendendo un albanese e spacciandolo per italiano": così Malaj ha provocatoriamente sintetizzato, non senza una nota di autocompiacimento, la spericolata iniziativa editoriale che lo ha visto protagonista ${ }^{7}$. Se per ben quattro volte durante gli anni Cinquanta lo Strega non fu assegnato ad un romanzo (grazie alle magistrali prove di Pavese, Moravia, Bassani e Buzzati sulla misura breve della narrazione), nelle seguenti edizioni si contano soltanto altre quattro eccezioni nell'arco dei sessant'anni fin qui trascorsi. Inoltre, nonostante una certa ripresa della produzione di genere in anni recenti, cui si accompagna un rinnovato fervore di studi critici e teorici ${ }^{8}$, sul mercato editoriale la raccolta di racconti continua ad essere vissuta come propedeutica all'esito romanzesco e a essere percepita come connaturata alla dimensione esordiale. Nel caso di Elvis Malaj però il romanzo, sebbene fin qui inedito, preesiste ai racconti, sennonché la raccolta ha trovato per una volta migliore accoglienza, segnale forse di quel nuovo interesse per il narrare breve che non ha mancato di produrre qualche

6. Lo stralcio della lettera si legge nel sito del Premio Strega: https://premiostrega. it/PS/elvis-malaj/.

7.Iltestointegraledell'intervistasilegge aquestoindirizzo:http://www.viadeiserpenti. it/racconti-italiani-2-intervista-a-elvis-malaj-un-cantastorie-che-diventa-scrittore/.

8. Si segnala almeno il seminario organizzato dall'Università di Bergamo con cadenza annuale: i recenti atti in Raccis Giacomo, SINFOnICO Domenico (a cura di), "Racconti di una vita", in Nuova corrente, n. 162, 15 novembre 2018; ZATTI Sergio, STARA, Arrigo (a cura di), "Un genere senza qualità. Il racconto italiano nell'epoca della short story", in Moderna, n. 2, 2010. 
effetto nel mondo delle riviste e della giovane editoria, in particolare in quella oggi vivacissima, on line e su carta, di area romana?.

Quanto fin qui annotato a proposito della pur breve carriera letteraria di Elvis Malaj, nella continua escursione tra sentire comune (minorità anagrafica, editoriale e di genere della candidatura) e dati di fatto (i precedenti di Giordano e Kubati, l'appoggio di Formenton, la recente voga della short story) può costituire un buon viatico per la lettura dei dodici racconti che formano l'architettura della sua prima opera edita. L'intera raccolta, infatti, invita il lettore a salire sull'ottovolante di storie che paiono precipitare verso conclusioni attese (sebbene non scontate) e, in quanto tali, gratificanti ma che inesorabilmente vanno incontro a svolte impreviste, magari in direzione della categoria narrativamente screditata del plausibile ${ }^{10}$, e a diversioni frustranti il talento predittivo di chi legge. Per intendersi, la medesima tecnica di cui è possibile citare un minimo campione traendolo però non dal testo ma dall'epitesto, ovvero da una dichiarazione rilasciata in un'intervista in merito al presunto significato dell'espatrio:

Ce l'hai presente quell'idea romantica del ricominciare? Andare alla stazione, salire su treno e partire, andare lontano, non importa dove, in una nuova città, una nuova vita, lasciarsi tutto alle spalle e ricominciare. Magari addirittura in un altro paese, imparare una lingua nuova, costruirti una vita tutta da capo, come piace a te, senza ripetere gli errori fatti. Abbandonare una vita logora e stantia in direzione di qualcosa di nuovo, ributtarsi nella mischia e cercare di cogliere l'occasione, avere il coraggio di salire su quel cazzo di treno perché la vita è solo tua e puoi farne quello che vuoi (la senti, la senti l'eccitazione?). Ecco, per me questa cosa non ha mai funzionato ${ }^{11}$.

9. Malaj è rappresentato dall'agenzia romana Oblique di Leonardo Luccone e ha pubblicato il suo primo racconto sulla rivista Effe, ospite del blog romano Flanerì, registrato come testata giornalistica presso il Tribunale di Roma. Notizia del romanzo già licenziato col titolo provvisorio di Il mare è rotondo è rimbalzata su vari siti, si veda ad esempio in questo blog della piccola editoria romana: http://www.viadeiserpenti.it/ racconti-italiani-2-lautobiografia-del-personaggio-che-poi-sarei-io/.

10. Come ha già notato Andrea Siviero, che ha intervistato Malaj per un blog tutto dedicato alle short stories: https://treracconti.it/intervista-elvis-malaj/.

11. Il brano è tratto dall'intervista rilasciata a Emanuela D'Alessio: http://www. viadeiserpenti.it/tag/elvis-malaj/. 
Fin dal titolo la raccolta invita ad assumere il punto di vista dell'altro o, almeno, a guadagnare una distanza dalla realtà del proprio vissuto tale da garantire lo spazio necessario all'atto intellettivo, alla comprensione, strategia che diventa prassi narrativa per un autore che fa mostra di governare con una certa spontaneità il tropo del distanziamento, l'ironia ${ }^{12}$. Il riferimento poi ad un accessorio condominiale come il terrazzo e alla sua dimensione dirimpettaia può richiamare alla mente uno dei titoli più fortunati della letteratura migrante, dove compariva quell" "ascensore" escogitato da Amara Lakhous come luogo della promiscuità coatta in una situazione urbana multietnica ${ }^{13}$. Paragone possibile a patto di rimodulare quella stridente promiscuità, che sfociava nell'omicidio, in una più mite prossimità, propizia alla love story. Del resto non mancano le ragioni per evidenziare come tra i racconti di Malaj e la nostra narrativa multiculturale dell'ultimo ventennio circoli una certa aria di famiglia (al netto di esplicite dichiarazioni di apprezzamento, come quella per la scrittura, eminentemente ironica, di Ornela Vorpsi) ${ }^{14}$, magari proprio a partire dall'adozione della forma breve che ha caratterizzato, per esempio, gli esiti più convincenti ed umoristici della prima stagione letteraria di Igiaba Scego ${ }^{15}$. Raffronti sono possibili col già citato e connazionale Ron Kubati, in particolare per quanto attiene al suo primo romanzo Va e non torna, dove si racconta della vita italiana di Elton e della relazione intrecciata a Bari con Elena, alternando spezzoni di vita albanese: le relazioni sentimentali tra un giovane albanese ed una ragazza italiana caratterizzano il primo e l'ultimo racconto della raccolta di Malaj, mentre altri narrano vicende balcaniche. Oppure con l'ormai lontana prima prova narrativa, e ultima, di Mihai Mircea Butcovan, alla quale la scrittura di Malaj si apparenta per certo sfoggio lessicale e, appunto, per la costante ironia: anche in Allunaggio di un immigrato innamorato si narrava di una sfortunata storia d'amore tra una giovane lombarda, e leghista, ed un giovane rumeno capace di aggiudicarsi in terra

12. Cfr. Pieranni Simone, "Elvis Malaj, riscrivere la distanza", in il manifesto, 6 dicembre 2017.

13. Il riferimento è qui a LaKHous Amara, Scontro di civiltà per un ascensore a piazza Vittorio, Roma, e/o, 2006.

14. "Ti dico la verità, pure la mia conoscenza della letteratura albanese è limitata. Ma un paio di nomi te li faccio: Gazmend Kapllani [...] e Ornela Vorpsi, perché sa scrivere": http://www.viadeiserpenti.it/ racconti-italiani-2-intervista-a-elvis-malaj-un-cantastorie-che-diventa-scrittore/.

15. Vale la pena di notare come, anche nella produzione romanzesca di Scego e di altri autori come Amara Lakhous, Gabriella Kuruvilla, Younis Tawfik ecc., prevalga una tecnica di costruzione modulare e combinatoria favorita da tecniche di narrazione plurifocale: cfr. FraCASSA Ugo, "Globalizzazione all'indice. Modelli macrotestuali nella narrativa dell'Italia multiculturale”, in Narrativa, n. 35-36, 2013-2014, pp. 101-111. 
padana il locale premio di poesia dialettale (il protagonista del racconto Dal tuo terrazzo si vede casa mia è anch'egli uno scrittore). La relazione tra un migrante e un'italiana mantiene insomma una sua centralità nel nuovo immaginario multiculturale, a conferma di quanto Ardian Vehbiu e Rando Devole ebbero a notare nell'ormai pionieristico studio sull'immagine degli albanesi veicolata dai mass media italiani nella prima metà degli anni Novanta: "il contatto sessuale tra un immigrato e una donna locale si presenta come estremamente carico di significati codificati e mitici"16.

Malaj è però innanzitutto uno scrittore autodidatta, quello che una volta si sarebbe detto un "narratore selvaggio", qualcuno che ammette di aver iniziato a leggere in italiano (il trasferimento dall'Albania risale al 2005), chiudendo prematuramente una ben avviata "carriera" di writer graffitista, e di aver divorato autori come Kafka, Tozzi, Svevo, Pirandello, Hesse prima di passare a letture disordinate che tuttavia non gli hanno impedito di incontrare l'opera di Carver, maestro del racconto e del dialogo, tecnica narrativa ampiamente dispiegata dal nostro. Circa l'esordio l'analogia è piuttosto col Tondelli di Altri libertini, folgorante debutto plasmato nel 1980 da Aldo Tagliaferri, editor feltrinelliano, a partire dall'incandescente materia di un brogliaccio romanzesco sesquipedale. Spettava a Tagliaferri, oltre che la fortunata titolazione del libello, il format narrativo scorciato in sei episodi a mo' di raccolta, così come a Leonardo Luccone, agente di Malaj, è forse riferibile la disposizione nell'indice dei racconti: "Sì, alla fine mi sono convinto anch'io che Vorrei essere albanese sia stata la scelta migliore [per aprire la raccolta]. Però, ti confesso, delle perplessità c'erano; questo è l'unico racconto di tutta la raccolta in cui il tema del razzismo viene affrontato" "17. Il libro del 2018 poi non è soltanto un esordio, ma un vero e proprio libro sugli esordi. I protagonisti dei racconti sono, infatti, degli absolute beginners, principianti, gente alle prime armi nello studio, nel lavoro, nell'arte, nell'amore, e le vicende che li riguardano si risolvono più spesso in false partenze che in capitoli di un fantomatico romanzo di formazione. Nell'ordine: una proposta di matrimonio abortita, una mancata deflorazione, un primo giorno di lavoro, un primo appuntamento, un primo giorno di scuola tutti variamente fallimentari e, da ultimo, un tentato suicidio utilitaristicamente sventato dalla proprietaria dell'auto parcheggiata sotto il balcone

16. Vehbiu Ardian, Devole Rando, La scoperta dell'Albania. Gli albanesi secondo i mass media, Milano, Paoline, 1996, p. 180.

17. Il brano è tratto dalla già citata intervista ad Andrea Siviero per il blog Tre racconti. 
del morituro (racconto nel racconto proveniente dal romanzo al quale il protagonista di Dal tuo terrazzo si vede casa mia sta lavorando).

Originalissimo nel debutto di Malaj l'utilizzo di prestiti non integrati dall'albanese fino all'inserimento di intere frasi in madrelingua. Tutto nasce da una deliberata o, quanto meno, coerente svalutazione del translinguismo ("Cosa vuol dire [esprimersi in una lingua diversa dalla propria]? Niente, fai diventare la nuova lingua la tua lingua"18) in un autore deciso a sottrarsi, e non senza ragione, alle soffocanti maglie della critica specializzata. Svalutazione che discende direttamente dall'attenuazione dell'enfasi comunemente posta sul vissuto migratorio, in particolare degli scrittori: "Trovarsi bene o meno in un posto non dipende dal posto, dipende da te. Ovunque vai ti porti sempre dietro qualcosa che alla fine rende ogni posto uguale all'altro"19. Questi racconti sono pieni di persone che non si comprendono pur parlando la stessa lingua, mentre altre colgono perfettamente il senso del discorso anche se proferito in una lingua straniera, definita talvolta "aspra" e "oscena" 20 . Il code-switching verso l'albanese operato da Marenglen nel primo racconto dimostra come, dovendo sortire un effetto minatorio, l'albanese suoni maggiormente dissuasivo alle orecchie di giovani bellunesi apparentemente desiderosi di menare le mani ("'Hiqi brrylat prej tavolins tonë, n'mos daç me ti thye dhëmbët'. Il ragazzo mi ha guardato perplesso e ha tolto i gomiti dal nostro tavolo. A volte l'albanese è più comprensibile dell'italiano"21). Al giovane protagonista di A pritni miq? capita invece di tornare all'albanese all'apice di un litigio con la compagna connazionale che lo ha seguito in Italia; se un simile comportamento trova precise spiegazioni nella psicolinguistica e risulta pertanto verosimile, il lettore è messo in condizioni di non comprendere nel dettaglio il contenuto del litigio che tuttavia, in quanto tale, rappresenta l'antonomasia dell'incomprensione (a litigare non ci si intende in nessuna lingua). È l'autore stesso, nell'Autobiografia del personaggio che poi sarei

18. L'intervista rilasciata ad Amelia D'Alessio si legge a questo indirizzo: http:// www.viadeiserpenti.it/racconti-italiani-2-intervista-a-elvis-malaj-un-cantastorie-che-diventa-scrittore/.

19. Malaj Elvis, Dal tuo terrazzo si vede casa mia, Roma, Racconti, 2017, p. 109.

20. Significativa, nel contribuire alla creazione di uno stereotipo globale, la scelta dell'albanese come antonomasia della lingua incomprensibile nel film Inside man di Spike Lee (2006), dove i rapinatori depistano gli investigatori grazie alle registrazioni dei discorsi di Enver Hoxha.

21. Malaj Elvis, Dal tuo terrazzo si vede casa mia, cit., p. 10. 
io, pur perfettamente bilingue, a non essere in grado di tradurre la lingua di sua madre, elemosinante in Caritas un pasto caldo, in italiano: “Ushqim' mi diceva mia madre, ma ero talmente imbarazzato che non sapevo più l'italiano" 22 . Eppure è proprio quando il ricorso alla madrelingua dovrebbe assicurare indecifrabilità che la comunicazione, malauguratamente, si riattiva per l'“albanesissimo" Kastriot, protagonista del racconto che dà il titolo al libro: "Ma c'è un albanese che non è ladro?' 'Stronza! [...] I ke sytë e bukur, i ke bythët e bukra, por nga nje herë je i çik kurvëë'. 'Hai detto kurv?' 'Perché, sai cosa vuol dire?' 'Ma guarda che bastardo! Mi hai dato della troia!"'23. Palese, dunque, l'intenzione metalinguistica nel seguente dialoghetto canino: "Rimasero un attimo a guardarsi, come marito e moglie pieni di rancore represso, poi il cane abbaiò un'altra volta. 'Non rompere i coglioni' diceva, da quello che capì Dedë. Stava parlando con un cane, pensò. 'Zot na ruaj' disse tra sé, e riprese a camminare"24, nel quale il lettore vede rappresentata in quella del personaggio la propria straniante esperienza. Al di là dell'apparente e, di primo acchito, urtante diversità, la presenza dello straniero può neutralizzare la propria "intraducibilità" se inserita in un contesto sociale accogliente, proprio come accade al prestito non integrato nel contesto della frase. In casi di integrazione, nella pragmatica linguistica o sociale, ogni differenza può farsi trasparente. Ben lungi dalle lusinghe dell'autonomia del significante insomma, gli inserti allofoni in Malaj configurano una riflessione sul linguaggio, certamente ambiziosa ma governata con mano ferma dallo scrittore debuttante. Si tratta ormai solo di aggiungere che lo specifico espediente linguistico seleziona e focalizza la condizione di chi è straniero come caso esemplare di quella fisiologica condizione di estraneità e spaesamento di chi, per ragioni anagrafiche, sia alla ricerca del suo posto nel mondo, condizione anch'essa specifica dei giovani e degli adolescenti (di Malaj) ma che può essere estesa a quella generica ed esistenziale di persona(ggio).

Nella frammentazione imposta dall'indice, nell'avvicendarsi di episodi narrativi ambientati ora al di qua ora al di là dell'Adriatico, non bisogna però sottovalutare la tensione verso una consistency, l'effetto cornice che innegabilmente

22. Si tratta di un testo pubblicato in rete nel quale Malaj ricapitola il proprio percorso esistenziale: https://www.illibraio.it/elvis-malaj-597630/.

23. Malaj Elvis, Dal tuo terrazzo si vede casa mia, cit., p. 160.

24. Ibid., p. 58. 
i dodici racconti producono ${ }^{25}$. Ed è altrettanto innegabile che la cornice consista proprio nella condizione di inbetweeness sperimentata dai personaggi in Dal tuo terrazzo si vede casa mia, titolo che può richiamare alla mente le parole di Edward Gibbon a proposito della nazione dirimpettaia del Bel Paese: "ben visibile dall'Italia ma meno conosciut[a] dell'interno dell'America" ${ }^{26}$. Non per questo Malaj pretende di parlare a nome di una comunità, ma fa suoi piuttosto i limiti di quei generi che, come la short story, "si vogliono parziali [...] e rinunciano ad ambizioni rappresentative"27. Del resto, esaurita la prima ondata dell'esodo migratorio culminato negli sbarchi del 1991, l'Albania è passata in breve tempo dalla rigida ortodossia isolazionista e puritana di Hoxha alla deregulation liberista inaugurata dal primo governo di Sali Berisha, da un'etica collettivista ad una individualista, ben riconoscibile anche nei modelli sociali delle fasi migratorie più recent ${ }^{28}$. E individualisti sono certamente i protagonisti di queste dodici storie, appena tradotte e pubblicate dalla maggiore casa editrice albanese. Tuttavia, a parere dell'autore che, richiesto di fornire un identikit del proprio lettore, taglia corto dicendo di figurarselo come "un tipo solitario", il libro (per il quale ha immediatamente rinunciato alla possibilità di auto-traduzione) rischia di andare incontro ad una ricezione problematica al di fuori del contesto italiano $^{29}$. Effettivamente il narrato delle dodici storie fa strutturalmente aggio su luoghi comuni, stereotipi e scorciatoie del pensiero in uso nella penisola, così tra gli autoctoni come tra i "nuovi italiani". Nell'Autobiografia del personaggio che poi sarei io Malaj confessa di aver appreso, coi primi rudimenti di civilizzazione italica, l'usanza dei nativi di gettare nel cassonetto varie masserizie ancora

25. Si tratta di quella "intenzione strutturale che [...] orienta le trame e crea correlazioni a distanza tra le totalità parziali delle diverse cellule di racconto" per la quale Silvia Acocella rimanda appunto alla nozione calviniana di consistency: AcocelLA Silvia, "Forma breve e forma lunga", in Il romanzo in Italia. Forme, poetiche, questioni, vol. III, Alfano Giancarlo, De Cristofaro Francesco (a cura di), Roma, Carocci, 2018, p. 142.

26. È Maria Todorova a citare le parole dello scrittore, scultore, storico e viaggiatore inglese di fine Settecento: Todorova Maria, Imagining the Balkans, Oxford, Oxford University Press, 2009 [1997], p. 45 [Lecce, Argo, 2002].

27. Guglielmi Guido, La prosa italiana del Novecento, Torino, Einaudi, 1986, p. 21. Analoga ma meno drastica la precedente ipotesi di Frank O'Connor, secondo il quale il racconto, non potendo rappresentare un insieme sociale più vasto e coeso, si specializzerebbe nella narrazione della vicenda umana di "submerged population groups" (O'Connor Frank, The Lonely Voice, London, McMillan, 1963, p. 18).

28. Cfr. Martelli Fabio, Capire l'Albania, Bologna, Il Mulino, 1998, pp. 210-211.

29. Traggo le dichiarazioni da una conversazione personalmente intrattenuta con Malaj il 9 dicembre 2018, a margine dell'annuale Salone della piccola e media editoria di Roma. 
fungibili, spunto che fornisce nel libro l'avvio per il racconto Il televisore $e^{30}$. Di fronte agli stereotipi però la passività non è l'unico atteggiamento possibile per il migrante che, se culturalmente attrezzato, può sovvertirli o piegarli ad un uso strategico. È quanto accade a "those migrants equipped with sufficient cultural capital" 31 , secondo quanto persuasivamente affermato da Stephanie Schwandner-Sievers in uno studio del 2008: "stereotypical imageries of Albanians [...] have forced contemporary transnational Albanian migrants into subversive strategies and practices of identity mimicry" ${ }^{\prime 32}$. Rispetto a simili tesi antropologiche i racconti di Elvis Malaj tollerano di essere utilizzati come materiale di esemplificazione letteraria. Il principale stigma imagologico applicato ai migranti provenienti dal paese delle aquile, quello di una violenza che troverebbe radici in remoti costumi tribali (ancora in uso nelle regioni montuose del nord dove resterebbe in vigore il codice consuetudinario del Kanun), è al centro del primo e dell'ultimo dei dodici episodi che compongono il volume. Nel primo caso, come visto, il giovane protagonista, perfettamente integrato e con tanto di fidanzata locale, fa ricorso alla lingua d'origine per terrorizzare gli imberbi avventori di un bar a Belluno. Nell'ultimo, viene ingaggiato da una ragazza italiana, di cui è invaghito, per ingelosire l'ex contro il quale, in virtù della sua albanesità, avrebbe certamente scatenato una rissa. Capita invece che sia l'italiano a colpire il malcapitato: "'Non posso farlo. Io sono per la non-violenza!' aveva dichiarato Kastriot. 'Ma è possibile che tra tutti gli albanesi che ci sono in giro proprio quello più sfigato dovevo scegliermi?"”33.

È stato notato che "il modo in cui gli autori di origine albanese parlano della propria terra natia si configura spesso in una narrativa che ha come fonte la memoria, la quale mette in atto un processo di mitologizzazione" ${ }^{34}$. Se questo è

30. "Non è detto che è rotto, può essere che l'hanno buttato perché ne hanno comprato un altro. Fanno così gli italiani, non sono come noi che prima telefoniamo a tutti i parenti, ai conoscenti, ai conoscenti dei parenti, per vedere se qualcuno lo vuole. Gli italiani lo buttano e basta": Malaj Elvis, Dal tuo terrazzo si vede casa mia, cit., p. 28.

31. Schwandner-Sievers Stephanie, "Albanians, Albanianism and the strategic subversion of stereotypes", in Anthropological notebooks, n. 14, 2008, p. 50.

32. Ibid., p. 47. Un esempio palmare di mimicry era già stato offerto da Amara Lakhous nel $2006 \mathrm{col}$ personaggio di Ahmed/Amedeo, protagonista dello Scontro di civiltà per un ascensore a piazza Vittorio.

33. Malaj Elvis, Dal tuo terrazzo si vede casa mia, cit., p. 138.

34. Shabaj Flora, Al di là e al di qua dell'Adriatico: la rappresentazione dell'Albania e dell'Italia tra $x \times$ e xxi secolo. Scrittori e scrittrici albanesi in lingua italiana a confronto, tesi 
vero, occorre aggiungere che l'immagine dei Balcani, e dunque anche quella dell'Albania, formatasi in Europa occidentale a partire dal XviII secolo e criticamente delineata, in quanto "balcanismo", da Maria Todorova, è stata rispecchiata e fatta propria al di là dell'Adriatico nel lungo processo di elaborazione delle identità nazionali, fino a produrre quella che è stata definita una "tradizione inventata" 35 . Un simile fenomeno di mirroring esperito dalle comunità fuori dai confini nazionali può trovare rappresentazione nella letteratura migrante e, per quanto attiene alla variante locale dell'“albanianesimo", nella narrativa di Elvis Malaj. Il racconto intitolato Scarpe, cui l'autore antepone la dedica "alla mia Albania" ${ }^{36}$, ne fornisce un valido esempio, giocato com'è sul registro di un carnevale balcanico à la Kusturica, tra la bagarre da bassa macelleria di un retrobottega e l'incontro carnale a buon mercato sul quale si chiude. Ciò che fa di Dal tuo terrazzo si vede casa mia un libro inclassificabile con le correnti categorie della critica è però il fatto che, a poche pagine da questo quadretto di genere, peraltro gustoso, ci si possa imbattere in un racconto come il crudele La carriola, pezzo di bravura per il quale l'unico riferimento possibile pare essere la turgida prosa di Federigo Tozzi alle prese con le sue Bestie:

Marash si chinò sulle lumache mezze conficcate nella terra e le contò. In tutto erano quattordici. [...] Con uno stecchino, Marash toccò il corpo molle della lumaca - tra il rosso e il marrone - che si versò fuori dal guscio. In un primo momento la lumaca cercò di fare presa sullo stecchino, ma poi all'improvviso rintanò. Marash creò una saccoccia con un lembo della maglietta, ci cacciò dentro le chiocciole e si piazzò davanti alla casa. Ne prese una e la scagliò contro il muro. Una roba gelatinosa bagnò i mattoni, e le schegge del guscio caddero per terra. Fece lo stesso con tutte le lumache, una a una ${ }^{37}$.

Ugo FraCASSA Università di Roma Tre

di dottorato in Scienze Linguistiche, Filologiche, Letterarie e Storico-Archeologiche, Università di Macerata, 2017, p. 183.

35. Cfr. Bardhoshi Nebi, "The Ethnography of Law in a Dictatorial Situation", in Boskovic A., Hann C. (a cura di), The Anthropological Field on the Margins of Europe, 1945-1991, Berlin, LIT Verlag, 2013.

36. Malaj Elvis, Dal tuo terrazzo si vede casa mia, cit., p. 49. Mio il corsivo.

37. Ibid., p. 83. 